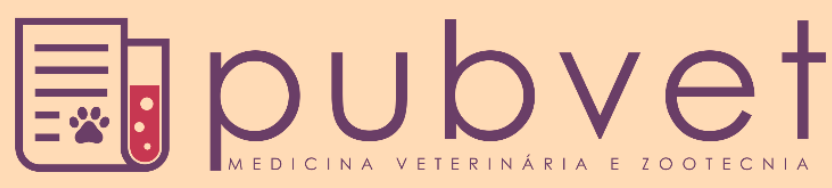

https://doi.org/10.31533/pubvet.v15n10a931.1-7

\title{
Temperatura de queijos Minas frescal expostos a venda em comércio varejista
}

\author{
Rafael Henrique de Mello $^{1} \bullet$, Alice Ferreira Drummond ${ }^{2} \bullet$, Bruna Maria Salotti de Souza ${ }^{3} \bullet$, Cláudia \\ Freire de Andrade Morais Penna ${ }^{3}{ }^{\circ}$, Marcelo Resende de Souza $^{3}{ }^{\circ}$, Elisa Helena Paz Andrade ${ }^{3 *} \bullet$ \\ ${ }^{1}$ Médico-veterinário da Prefeitura de São Gabriel da Palha, Departamento de Vigilância em Saúde. São Gabriel da Palha - ES, Brasil. \\ ${ }^{2}$ Médica-veterinária do Instituto de Defesa Agropecuária e Florestal do Espírito Santo (IDAF), Departamento de Defesa Animal. Alegre-ES, Brasil. \\ ${ }^{3}$ Professor da Universidade Federal de Minas Gerais (UFMG), Departamento de Tecnologia e Inspeção de Produtos de Origem Animal. Belo Horizonte - MG, Brasil. \\ *Autor para correspondência, E-mail: elisahpandrade@yahoo.com.br
}

Resumo. Esta pesquisa teve por objetivo investigar as temperaturas de queijos Minas frescal, um dos derivados lácteos mais consumidos no Brasil, em estabelecimentos comercializadores, com o intuito de verificar a adequação desse parâmetro com o preconizado pela legislação pertinente e com o que era estabelecido nos rótulos. Para tanto, foram aferidas temperaturas de 50 peças de queijo Minas frescal em dez supermercados, localizados no município de São Gabriel da Palha, Espírito Santo. Foi ainda tomado nota do tipo de equipamento utilizado para acondicionamento desses queijos em cada um dos estabelecimentos e da temperatura máxima especificada nos rótulos para conservação. Os resultados foram então confrontados com a temperatura máxima de conservação especificada no rótulo e com o que é determinado pela legislação. Foi constatado que o equipamento utilizado para exposição dos queijos sob refrigeração era do tipo vitrine, variando entre os modelos de vitrines aberta (40\%) e fechada (60\%). Foi observado que $60 \%$ dos estabelecimentos de venda mantinham os queijos conservados de acordo com a temperatura estipulada pelo fabricante e pela legislação e $83,3 \%$ desses estabelecimentos empregavam o modelo de equipamento de conservação a frio do tipo vitrine fechada. Esse achado sugere que este modelo proporciona um controle da temperatura de conservação mais efetivo, sendo mais indicado para acondicionamento de queijo Minas frescal. Os estabelecimentos comercializadores devem, portanto, adotar medidas para garantir que os queijos Minas frescal permaneçam acondicionados em temperatura adequada e os órgãos fiscalizadores devem intensificar a vigilância desses produtos expostos a venda.

Palavras chave: Conservação, derivado lácteo, estabelecimentos de venda, rótulo, vitrine fechada

\section{Temperature of fresh Minas cheeses exposed for sale in retail trade}

Abstract. This research aimed to investigate the temperatures of fresh Minas cheeses, one of the most consumed dairy products in Brazil, in commercial establishments, with the objective of verifying the adequacy of this parameter with that recommended by the legislation and with what was described on the labels. For this purpose, temperatures of 50 samples of fresh Minas cheese were measured in ten supermarkets, located in the municipality of São Gabriel da Palha, Espírito Santo. Notes were also taken of the type of equipment used to store the cheeses in each establishment and the maximum storage temperature specified on their labels for the conservation. The results were compared with the maximum storage temperature specified on the label and with what is determined by the legislation. It was found that the equipment used to display the cheeses under refrigeration was showcase type, varying between the opened (40\%) and closed (60\%) models. It was observed that $60 \%$ of the sale establishments kept the cheeses preserved according to the temperature recommended by the manufacturer and by the legislation and 
$83.3 \%$ of them used the closed showcase type. This finding suggests that this model provides a more effective control of the storage temperature, being more suitable for the conservation of fresh Minas cheese. The commercial establishments must, therefore, adopt measures to ensure that the cheeses remain stored at an adequate temperature and the inspection agencies must intensify the surveillance of these products exposed for sale.

Keywords: Conservation, dairy product, sale establishments, label, closed showcase

\section{Temperatura de los quesos Minas frescos expuestos a la venta en el comercio minorista}

Resumen. Esta pesquisa tuvo por objetivo averiguar las temperaturas de quesos Minas fresco, uno de los productos lácteos más consumidos en Brasil, en establecimientos comerciales, con la intención de conocer la adecuación de este parámetro con el preconizado de la legislación pertinente y el establecido en la etiqueta. Por tanto, fueron referidas las temperaturas de 50 piezas de queso Minas fresco en diez supermercados, ubicados en la provincia de São Gabriel da Palla, Espírito Santo. También se toma nota de todo el equipamiento utilizado para el condicionamiento de los quesos en cada uno de los establecimientos y la temperatura máxima especificada en las etiquetas para conservación. Los resultados son confrontados con la temperatura máxima de conservación especificada en la etiqueta y la determinada por la legislación. Fue constatado que el equipo utilizado para la exposición de los quesos en refrigeración son del tipo escaparate, variando entre los modelos de escaparate abierto (40\%) y cerrado (60\%). Se observa que $60 \%$ de los establecimientos de venta mantiene los quesos conservados de acuerdo con la temperatura estipulada por el fabricante y por la legislación, y 83,3\% de estos establecimientos empleaban el modelo de equipo de conservación a frío del tipo escaparate cerrado. Este hallazgo sugiere que este modelo proporciona un control de la temperatura de conservación más efectivo, siendo más indicado para condicionamiento del queso Minas fresco. Los establecimientos comercializadores deben, sin embargo, adoptar medidas para garantizar que los quesos Minas fresco permanezcan condicionados en la temperatura adecuada y los órganos fiscalizadores deben intensificar la vigilancia de estos productos expuestos a la venta.

Palabras clave: Conservación, derivados lácteos, establecimientos de venta, etiqueta, escaparate cerrado

\section{Introdução}

Queijo Minas Frescal é o "queijo fresco obtido por meio da coagulação enzimática do leite pasteurizado com coalho ou com outras enzimas coagulantes apropriadas ou com ambos, complementada ou não pela ação de bactérias lácticas específicas, com a obtenção de uma massa coalhada, dessorada, não prensada, salgada e não maturada" (Brasil, 2017). Por possuir elevado teor de umidade, esse produto é altamente perecível. Além disso, durante sua fabricação, o queijo Minas frescal passa por muitas etapas de manipulação, o que proporciona condições ideais para contaminação, sobrevivência e proliferação de bactérias e fungos, que podem ser patogênicos ou produtores de metabólitos causadores de intoxicações alimentares (Amorim et al., 2014; Apolinário et al., 2014; Câmara et al., 2002; Castro et al., 2013; Garcia et al., 2017; Trombete et al., 2012).

Agroindústrias produtoras de queijo Minas frescal licenciadas pelos órgãos regulamentadores competentes devem obedecer a uma série de exigências estipuladas pela legislação, para garantir a qualidade e a segurança do alimento a ser consumido (Visotto et al., 2011). As legislações de rotulagem de produtos de origem animal estabelecem a obrigatoriedade da especificação da forma de conservação no rótulo para que esta seja seguida durante as etapas de transporte, armazenamento e comercialização (Brasil, 2002; Brasil, 2005a; Brasil, 2017).

O Regulamento Técnico Mercosul de Identidade e Qualidade (RTIQ Mercosul) do Queijo Minas Frescal estabelece que, para sua conservação e comercialização, o produto não deve ser mantido a temperatura superior a $8^{\circ} \mathrm{C}$ (Brasil, 1996). O Regulamento de Inspeção Sanitária e Industrial de Leite e 
Derivados determina que a temperatura de armazenamento do queijo Minas frescal não deve ser superior a $7^{\circ} \mathrm{C}$ (Brasil, 2005b).

Tendo em vista o que foi exposto, o presente trabalho teve como objetivo avaliar a temperatura de queijos Minas frescal expostos no comércio varejista de São Gabriel da Palha, Espírito Santo, para verificar conformidade com a legislação e com o que era estipulado nos rótulos desses produtos.

\section{Material e métodos}

A pesquisa foi realizada em São Gabriel da Palha, município situado na região noroeste do Espírito Santo. São Gabriel da Palha possui extensão territorial de $434,88 \mathrm{Km}^{2}$ e população estimada de 38.522 habitantes em 2020 (IBGE, 2020).

Os dados foram obtidos de dez empresas da área de supermercados, todas com descrição de atividade "Comércio varejista de mercadorias em geral com predominância em alimentos - Supermercados", de acordo com a Classificação Nacional de Atividades Econômicas (CNAE) (IBGE, 2021).

Para cada estabelecimento incluído no estudo, foi tomado nota do tipo de equipamento utilizado para acondicionamento dos queijos Minas frescal. As mensurações de temperatura foram realizadas levandose em consideração os produtos expostos para consumo.

Os valores de temperaturas foram obtidos de peças de queijos Minas frescal inteiras provenientes de estabelecimentos sob fiscalização do Serviço de Inspeção Estadual (SIE) ou do Serviço de Inspeção Municipal (SIM). A coleta de dados foi realizada em um único dia do mês de abril de 2018, em um período de três horas compreendido entre 10 e 13 horas, evitando, assim, alterações de clima que pudessem influenciar indiretamente a temperatura dos produtos avaliados.

As temperaturas foram aferidas utilizando Termômetro Digital Mira Laser - 30 a $550^{\circ} \mathrm{C}$ com emissividade Pré-ajustada Minipa ${ }^{\circledR}$ MT-350 (Texas, Estados Unidos da América), previamente calibrado. O laser foi incidido diretamente sobre a peça de queijo, com o aparelho mantido a uma distância de $15 \mathrm{~cm}$. As temperaturas obtidas foram lidas em graus Celsius pelo visor do termômetro e anotadas diretamente em tabela de registro. Em cada supermercado, foram aferidas as temperaturas de cinco unidades de queijo de uma única marca localizadas em diferentes posições dentro do equipamento de refrigeração. Foi também anotada a temperatura máxima de conservação indicada no rótulo pelo fabricante de cada produto para posterior verificação de conformidade.

Para as análises estatísticas dos resultados obtidos na pesquisa, foram considerados os resultados individuais colocados em tabela, incorporada ao programa Excel ${ }^{\circledR}$. Com o auxílio desse programa, foram calculados as médias aritméticas e os desvios-padrões dos valores de temperatura registrados nas peças de queijo em cada um dos estabelecimentos.

\section{Resultados e discussão}

Nesse estudo, 50 peças inteiras de queijo Minas frescal tiveram sua temperatura verificada. Os equipamentos de conservação a frio utilizados para exposição dos queijos eram do tipo vitrine, variando entre os modelos de vitrines aberta e fechada. Dos dez supermercados visitados, quatro (40\%) possuíam equipamento de frio do tipo vitrine aberta e seis (60\%) do tipo vitrine fechada (Figura 1).

As médias de temperaturas encontradas para os queijos Minas frescal bem como o desvio padrão por estabelecimento segundo o tipo de vitrine utilizada e a temperatura máxima de armazenamento indicada no rótulo são apresentados na Tabela 1. Foi possível observar que, dos dez estabelecimentos varejistas incluídos nesta pesquisa, seis $(60 \%)$ mantinham seus produtos dentro dos padrões de temperatura de conservação estabelecidos pelo fabricante, respeitando as legislações vigentes $\left(<7^{\circ} \mathrm{C}\right)$. Desses seis, cinco $(83,3 \%)$ apresentavam equipamento de conservação a frio do tipo vitrine fechada.

Considerando os estabelecimentos que mantinham os queijos Minas frescal em equipamentos de conservação a frio tipo vitrine aberta, em um deles (25\%) foi encontrada a temperatura média de $6,5^{\circ} \mathrm{C}$, estando essa conforme estabelecido na rotulagem do produto, no RTIQ Mercosul e no Regulamento de Inspeção Sanitária e Industrial para Leite e Derivados. Nos demais estabelecimentos com esse tipo de equipamento de conservação a frio, os produtos apresentaram-se com temperaturas superiores a $7,0^{\circ} \mathrm{C}$ (Tabela 1). 
Cinco dos seis estabelecimentos $(83,3 \%)$ que mantinham os queijos Minas frescal em equipamentos de conservação a frio do tipo vitrine fechada apresentaram temperatura inferior ao indicado na rotulagem do produto e conforme estabelecido no RTIQ Mercosul e no Regulamento de Inspeção Sanitária e Industrial para Leite e Derivados (Tabela 1). Ademais, os queijos Minas frescal acondicionados nesse tipo de equipamento também demonstraram menor variação da temperatura de refrigeração, considerando produtos dispostos em posições diferentes dentro da vitrine. Esses achados sugerem maior efetividade desse modelo de equipamento no controle da temperatura. Isso pode ser explicado pela barreira física que o vidro da vitrine exerce sobre o frio, evitando a troca de temperatura do aparelho com a atmosfera do ambiente, facilitando a manutenção e preservação da temperatura em seu interior.
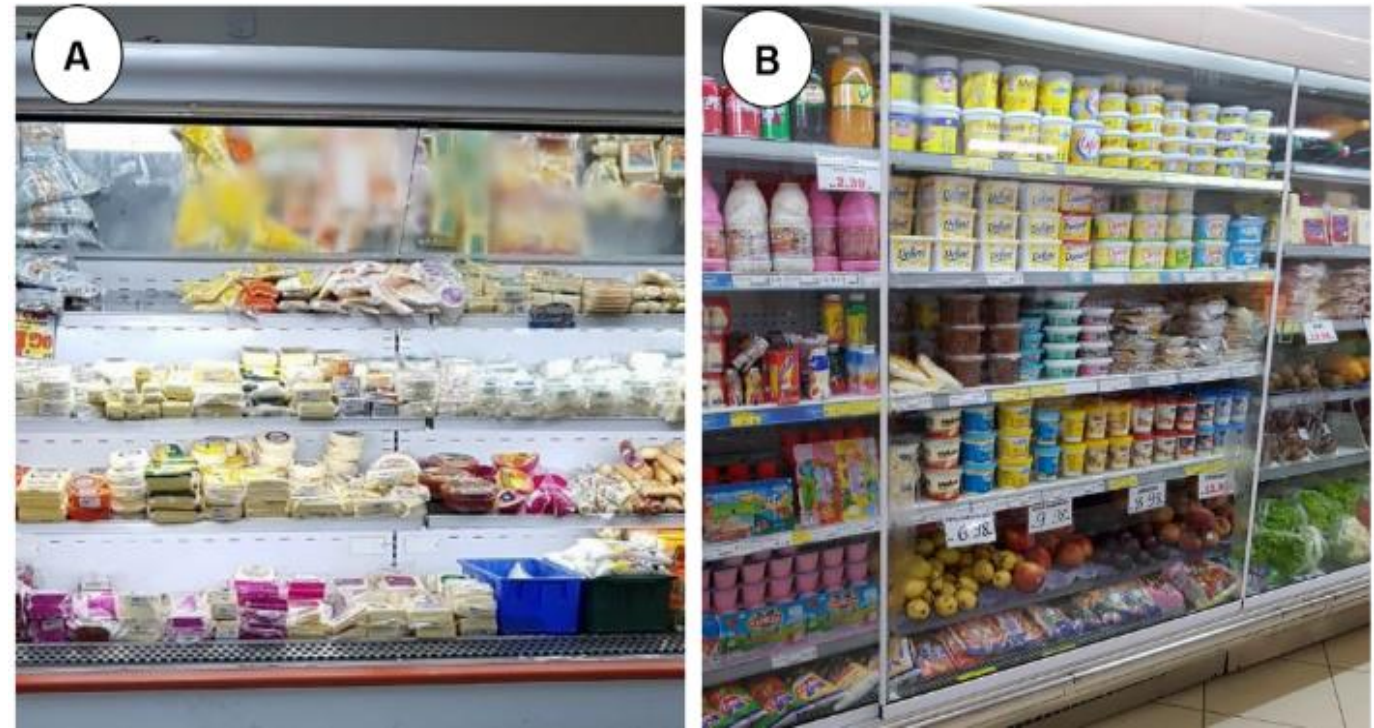

Figura 1. Modelos de equipamento de conservação a frio utilizados para acondicionamento de produtos refrigerados expostos a venda em dois supermercados do estudo em São Gabriel da Palha, Espírito Santo: (A) tipo vitrine aberta e (B) tipo vitrine fechada.

Tabela 1. Valores médios de temperatura de queijos Minas frescal e desvios padrões obtidos em cada estabelecimento do estudo de acordo com o tipo de vitrine utilizada e temperatura máxima indicada no rótulo de cada produto.

\begin{tabular}{|c|c|c|c|}
\hline \multirow{2}{*}{ Supermercado } & \multirow{2}{*}{ Tipo De Vitrine } & \multicolumn{2}{|c|}{ Temperatura Queijo Minas Frescal } \\
\hline & & Média obtida \pm DP* & Máxima indicada no rótulo \\
\hline 1 & \multirow{4}{*}{ Aberta } & $6,5 \pm 1,2^{\circ} \mathrm{C}$ & Até $7^{\circ} \mathrm{C}$ \\
\hline 2 & & $7,5 \pm 1,0^{\circ} \mathrm{C}$ & Até $7{ }^{\circ} \mathrm{C}$ \\
\hline 3 & & $8,0 \pm 2,5^{\circ} \mathrm{C}$ & Até $7{ }^{\circ} \mathrm{C}$ \\
\hline 4 & & $10,5 \pm 1,5^{\circ} \mathrm{C}$ & Até $7{ }^{\circ} \mathrm{C}$ \\
\hline 5 & \multirow{6}{*}{ Fechada } & $7,5 \pm 0,3^{\circ} \mathrm{C}$ & Até $7^{\circ} \mathrm{C}$ \\
\hline 6 & & $5,0 \pm 0,2^{\circ} \mathrm{C}$ & Até $7^{\circ} \mathrm{C}$ \\
\hline 7 & & $4,5 \pm 0,5^{\circ} \mathrm{C}$ & Até $8^{\circ} \mathrm{C}$ \\
\hline 8 & & $6,5 \pm 0,5^{\circ} \mathrm{C}$ & Até $7{ }^{\circ} \mathrm{C}$ \\
\hline 9 & & $6,0 \pm 0,2^{\circ} \mathrm{C}$ & Até $7{ }^{\circ} \mathrm{C}$ \\
\hline 10 & & $5,5 \pm 0,5^{\circ} \mathrm{C}$ & Até $7{ }^{\circ} \mathrm{C}$ \\
\hline
\end{tabular}

*Desvio-padrão

Lúcio et al. (2020) avaliaram a temperatura de derivados lácteos (manteiga, requeijão, creme de leite e leite pasteurizado) expostos a venda em refrigeradores com vitrine fechada, em um hipermercado localizado no interior do estado de São Paulo. A avaliação de temperatura foi realizada em três horários diferentes $(9,12$ e $15 \mathrm{~h})$. Os autores observaram que as temperaturas dos produtos lácteos analisados se apresentavam dentro dos padrões estabelecidos pela legislação vigente e de acordo com a temperatura de conservação especificada no rótulo. Além disso, não houve diferença significativa nas mensurações realizadas em diferentes períodos do dia. Assim como foi observado no presente trabalho, o emprego de refrigeradores do tipo vitrine fechada pode ter contribuído para a manutenção da temperatura dos produtos adequada. 
O armazenamento correto dos alimentos é um fator fundamental para manutenção de sua qualidade, de forma que condições satisfatórias de controle de temperatura devem ser sempre observadas para garantir e manter os padrões higiênico-sanitários dos produtos. Quando armazenados em temperaturas inadequadas, os alimentos em geral podem sofrer alteração de suas características sensoriais e aumento da suscetibilidade a contaminação por microrganismos patogênicos (

As doenças transmitidas por alimentos (DTA) constituem um dos problemas de saúde pública mais frequentes do mundo contemporâneo, podendo ser causadas pela ingestão de alimentos e/ou água contaminados por bactérias e suas toxinas, vírus e outros parasitas (Brasil, 2020). Segundo a Secretaria de Vigilância em Saúde (SVS) do Ministério da Saúde, leite e derivados estão entre os principais alimentos incriminados em surtos de DTA, de acordo com um levantamento realizado de 2007 a 2018 (Brasil, 2019).

O leite e seus derivados, como o queijo Minas Frescal, apresentam características intrínsecas favoráveis para multiplicação de micro-organismos, como a sua própria composição e elevada atividade de água, o que os torna um meio de cultura ideal para crescimento microbiano. Dessa forma, quando esses produtos são mantidos em temperatura de refrigeração controlada, é possível promover a manutenção de suas características sensoriais e microbiológicas (Sadhu, 2018).

No presente trabalho, quatro (40\%) dos dez estabelecimentos visitados apresentaram equipamentos de frio com temperatura inadequada para acondicionamento dos queijos Minas frescal, todos com vitrine do tipo aberta. Dias et al. (2016) verificaram que as temperaturas de duas amostras de queijo Minas frescal industrializado dentre cinco avaliadas em supermercado da região Sul de Goiás eram de 11,1 e $11,3^{\circ} \mathrm{C}$, superiores ao preconizado pelo Regulamento de Inspeção Sanitária e Industrial para Leite e Derivados e pelo RTIQ Mercosul. Todas as amostras de queijo Minas frescal artesanal coletadas nesse estabelecimento de venda apresentaram temperatura maior que $8^{\circ} \mathrm{C}$, variando entre 9,9 e $21,8^{\circ} \mathrm{C}$. Em um estudo conduzido por Lopes et al. (2020), a temperatura de queijos Minas frescal foi aferida em supermercados do município de Belo Horizonte, Minas Gerais. Oito $(88,9 \%)$ das nove amostras de queijo Minas frescal industrializados avaliadas se encontravam em temperatura acima do permitido. Os autores consideraram que a exposição a venda sob temperaturas inadequadas pode ter contribuído para as altas contagens microbianas encontradas naqueles produtos.

Os equipamentos utilizados para conservação de produtos perecíveis no momento da comercialização, como balcões refrigerados, muitas vezes não atendem aos padrões exigidos de temperatura. Assim, se tornam um ponto que requer atenção, pois a manutenção da temperatura de refrigeração pode prevenir, reduzir ou eliminar os riscos de ocorrência de DTA, principalmente às de origem microbianas (Arruda et al., 1996). Dessa forma, os estabelecimentos comercializadores devem adotar medidas para garantir que os queijos Minas frescal permaneçam acondicionados em temperatura adequada e os órgãos fiscalizadores devem intensificar a vigilância da temperatura desses produtos expostos a venda.

Algumas ações podem contribuir para a manutenção da temperatura adequada no equipamento de conservação a frio: controlar a temperatura do ambiente, instalar termostato no equipamento de frio, mantê-lo devidamente regulado e realizar a higienização do mesmo regularmente (Sousa et al., 2003). Foi possível observar que alguns estabelecimentos de venda incluídos no estudo apresentavam ambiente climatizado, mas isso não foi padrão entre os supermercados.

Cabe ressaltar que há necessidade de serem oferecidas orientações e capacitações periódicas aos colaboradores responsáveis pelo setor de resfriados dos estabelecimentos de venda, em relação às condições de manipulação e conservação desses produtos, seja por parte da empresa ou por parte dos órgãos públicos. É de grande importância que esses colaboradores estejam cientes da real importância de se conservar esses produtos em condições ideais e sejam treinados para a identificação de possíveis erros de armazenamento, a adoção de ações corretivas e o monitoramento frequente de parâmetros como a temperatura, com o intuito de mitigar o risco da ocorrência de produtos com a temperatura superior ao preconizado. 


\section{Conclusão}

Considerando os estabelecimentos incluídos no estudo, $60 \%$ mantinham os queijos Minas frescal dentro dos padrões de temperatura de conservação estabelecidos pelo fabricante e de acordo com as legislações vigentes. O modelo de equipamento de conservação de frio empregado por 83,3\% desses estabelecimentos foi o de vitrine fechada. Portanto, sugere-se que este tipo de equipamento de conservação de frio proporcione manutenção mais eficiente da temperatura quando comparado ao modelo de vitrine aberta, sendo o mais indicado para a exposição de queijos Minas frescal.

\section{Referências bibliográficas}

Amorim, A. L. B. C., Couto, E. P., Santana, A. P., Ribeiro, J. L., \& Ferreira, M. de A. (2014). Avaliação da qualidade microbiológica de queijos do tipo Minas padrão de produção industrial, artesanal e informal. Revista do Instuto Adolfo Lutz, 73(4), 364-367.

Apolinário, T. C. C., Santos, G. S., \& Lavorato, J. A. A. (2014). Avaliação da qualidade microbiológica do queijo Minas frescak produzido por latícinios do estado de Minas Gerais. Revista do Instituto de Laticínios Cândido Tostes, 69(6), 433-442.

Arruda, G. A., Popolim, W. D., Fujino, H., Leite, C. L., \& Ribeiro, L. C. (1996). Avaliação das condições de entrega de gêneros perecíveis em unidades de alimentação e nutrição, através do método de análise de perigos em pontos críticos de controle (APPCC). Higiene Alimentar, 10(44), 44-48.

Brasil. Decreto ${ }^{\circ} 9013$, de 29 de março de 2017. Regulamenta a Lei $n^{\circ} 1.283$, de 18 de dezembro de 1950, e a Lei $n^{\circ} 7.889$, de 23 de novembro de 1989, que dispõem sobre a inspeção industrial e sanitária de produtos de origem animal. Brasília: Ministério da Agricultura, Pecuária e Abastecimento. 2017.

Brasil. Ministério da Agricultura, Pecuária e Abastecimento. Instrução Normativa 22, de 24 de novembro de 2005. Regulamento Técnico para Rotulagem de Produtos de Origem Animal Embalado. Brasília, 2005a.

Brasil. Ministério da Agricultura, Pecuária e Abastecimento. Regulamento da Inspeção Sanitária e Industrial para Leite e seus Derivados. Brasília, $2005 \mathrm{~b}$.

Brasil. Ministério da Saúde. Agência Nacional de Vigilância Sanitária. Resolução - RDC n. 259, de 20 de setembro de 2002. Regulamento Técnico sobre Rotulagem de Produtos Embalados. Brasília, 2002.

Brasil. Resolução Mercosul/GMC/RES.Nº145/96 de 13 de dezembro de 1996. Regulamento Técnico Mercosul de Identidade e Qualidade de Queijo Minas Frescal do Ministério da Agricultura, Pecuária e Abastecimento. Diário Oficial da República Federativa do Brasil, Brasília, 1996. Disponível em: <http://www.inmetro.gov.br/barreirastecnicas/PDF/GMC_RES_1996-145.pdf>. Acesso em 08 jun. 2018.

Brasil. Ministério da $2019 . \quad$ Daúde, em: <https://antigo.saude.gov.br/images/pdf/2019/maio/17/Apresentacao-Surtos-DTA-Maio-2019.pdf>. Acesso em 20 jul. 2019.

Brasil. Ministério da Saúde, 2020. Disponível em: <http://antigo.saude.gov.br/saude-de-a-z/doencastransmitidas-por-alimentos>. Acesso em 11 nov. 2020.

Câmara, S. A. V., Muller, M. T., Silveira, K. C. S., Almeida, T. N., \& Medeiro, C. F. (2002). Avaliação microbiológica de queijos tipo Minas frescal artesanal, comercializados no mercado municipal de Campo Grande, Mato Grosso do Sul, 2000. Higiene Alimentar, 101, 32-36.

Castro, A. C. S., Pinto Júnior, W. R., Tápia, D. M. T., \& Cardoso, L. G. V. (2013). Avaliação da qualidade físico-química e microbiológica de queijos do tipo mussarela comercializados no ceasa de Vitória da Conquista - BA. Alimentos e Nutrição Araraquara, 23(3), 414.

Dias, B. F., Ferreira, S. M., Carvalho, V. S., \& Soares, D. S. B. (2016). Qualidade microbiológica e físico-química de queijo minas frescal artesanal e industrial. Revista de Agricultura Neotropical, 3(3), 57-64.

Garcia, E. P., Silva, F. A. R., Paiva Filho, O. M., Silva, D. H. L., Braga, A. V. U., Morelli, S. A., \& Santos, R. F. S. (2017). Qualidade microbiológica de queijos minas frescal e ricota comercializados na Região Metropolitana de Campinas-SP. Higiene Alimentar, 264-265, 132-137. 
Instituto Brasileiro de Geografia e Estatística (IBGE). Cidades. 2020. Disponível em: $<$ https://www.ibge.gov.br/cidades-e-estados/es/sao-gabriel-da-palha.html>. Acesso em 27 de janeiro de 2021.

Instituto Brasileiro de Geografia e Estatística (IBGE). Comissão Nacional de Classificação, 2021. Disponível em: $<$ https://cnae.ibge.gov.br/?view=estrutura\&tipo $=$ cnae\&versao_classe $=7.0 .0 \&$ versao_subclasse=9.1.0. $>$. Acesso em 13 de fevereiro de 2021.

Lopes, V. C., Guedes, E. K., Candioto, M. V. C., Delvivo, F. M., \& Lima, A. R. (2020). Qualidade microbiológica de queijos tipo Minas comercializados em Belo Horizonte, MG, Brasil. InfarmaCiências Farmacêuticas, 32(4), 344-352.

Lúcio, A. F., Gomes, J. E. G., \& Souza, B. M. S. (2020). Avaliação da temperatura de produtos lácteos expostos em unidades produtoras de frio de hipermercado. Higiene Alimentar, 34(290), 32-39.

Sadhu, S. P. (2018). Effect of cold chain interruptions on the shelf-life of fluid pasteurised skim milk at the consumer stage. Brazilian Journal of Food Technology, 21, 1-9.

Santos, T. P., Pinheiro, R. E. E., \& Júnior, M. H. K. (2015). Análise da rotulagem de produtos cárneos comercializados em Teresina, Piaú. Revista Brasileira de Higiene e Sanidade Animal, 9(3), 364-379.

Sousa, C. L., Faria, C. P., \& Neves, E. C. A. (2003). Avaliação da temperatura de balcões e câmaras frias de armazenamento de queijos e embutidos em supermercados da cidade de Belém-PA (Brasil). Boletim Do Centro de Pesquisa de Processamento de Alimentos, 21(1).

Trombete, F. M., Fraga, M. E., \& Saldanha, T. (2012). Avaliação da qualidade química e microbiológica de queijo parmesão ralado comercializado no Rio de Janeiro. Revista do Instituto e Laticinios Cândido Tostes, 67(385), 11-16.

Visotto, R. G., Oliveira, M. A., Prado, S. P. T., \& Bergamini, A. M. M. (2011). Queijo Minas Frescal: perfil higiênico-sanitário e avaliação da rotulagem. Revista do Instituto Adolfo Lutz, 70(1), 8-15.

Histórico do artigo:

Recebido: 10 de maio de 2021

Aprovado: 28 de junho de 2021
Licenciamento: Este artigo é publicado na modalidade Acesso Aberto sob a licença Creative Commons Atribuição 4.0 (CC-BY 4.0), a qual permite uso irrestrito, distribuição, reprodução em qualquer meio, desde que o autor e a fonte sejam devidamente creditados. 